\title{
QUELQUES RÉFLEXIONS AUTOUR DU PROJET DE LOI FRANÇAIS RELATIF À LA BIODIVERSITÉ
}

Domien Thierry*

Annoncé depuis longtemps, le «projet de loi relatif à la biodiversité» doit être discuté au cours de cet été 2014 devant le parlement français afin de moderniser la politique de conservation de la faune, de la flore et des habitats en France près de quarante ans après la loi du 10 juillet 1976 sur la protection de la nature ${ }^{1}$ qui en a posé les premières bases.

Ce projet s'appuie sur un constat initial, celui des grandes responsabilités françaises en la matière dans la mesure où il ressort des derniers bilans que seulement $22 \%$ des habitats et $28 \%$ des espèces d'intérêt communautaire en France sont en bon état de conservation? ${ }^{2}$. Ces chiffres alarmistes justifient à eux seuls une reprise en main de cette politique. Par ailleurs, le projet est présenté comme répondant à une évolution d'ordre social en ce sens que les Français apparaîtraient aujourd'hui de plus en plus sensibles aux questions touchant à la biodiversité. Les Français manifesteraient ainsi leur préoccupation non seulement devant l'érosion de la biodiversité mais iraient jusqu'à considérer que cette perte a un impact sur leur quotidien ${ }^{3}$. Enfin, cette réforme s'inscrit dans un mouvement qui a permis de faire évoluer la conception de la protection. Au fil du temps on est en effet passé d'une logique de

\footnotetext{
* Maître de conférences en droit public à l'Université F. Rabelais de Tours Membre du Groupe d'étude et de recherche sur la coopération internationale et européenne [GERCIE, EA 2110)

1 Loi n $76-629$ du 10 juillet 1976 relative à la protection de la nature.

2 Chiffres cités dans l'exposé des motifs accompagnant le projet de loi sur la biodiversité.

${ }^{3}$ «Les français et la bjodiversité» - Enquête CREDOC 2013 citée dans l'exposé des motifs accompagnant le projet de loi sur la biodiversité.
} 
protection des espèces vers une protection des espaces et des milieux dans lesquels elles évoluent. Puis prenant conscience que cette politique risque de produire un mitage de la nature, un échantillonnage d'espaces protégés noyés dans un environnement général très dégradé, l'action publique s'est ensuite diversifiée pour prendre en compte des aspects de plus en plus en complexes tels que les continuités écologiques représentées notamment par la «Trame verte et bleue» qui défend l'idée de corridors écologiques afin de maintenir un lien entre les périmètres préservés ${ }^{4}$.

Ce projet de loi est cependant loin de répondre aux nombreux enjeux posés par la préservation de la biodiversité en France. Quantitativement important, avec ses 72 articles organisés en 6 titres, il comprend en réalité toute une série de mesures dont l'intérêt est très variable. Si un certain nombre d'innovations sont notables, beaucoup de réformes demeurent accessoi. res ou incomplètes, voire éloignées du sujet principal. II en est ainsi des dispositions concernant la réforme des parcs naturels régionaux ${ }^{5}$ ou du régime des sites et paysages ${ }^{6}$ dont on voit mal en quoi elles vont avoir un impact sur la biodiversité. Enfin, le projet comprend un large volet consacré à l'espace marin qui ne sera pas abordé ici?

L'objet de la présente étude n'est pas d'aborder l'ensemble des points inscrits dans le projet de loi, mais a pour modeste ambition d'en relever quelques aspects. II s'agira tout particulièrement de s'intéresser à la réforme du cadre général qui tend à améliorer la vision d'ensemble de la biodiversité $[1$ ] et de s'arrêter sur quelques dispositions concrètes qui tendent à assurer une prise en compte effective de la biodiversité (II].

\section{I - LE CADRE GÉNÉRAL: AMÉLIORER LA VISION D'ENSEMBLE DE LA BIO. DIVERSITÉ}

En déposant ce projet de loi, le ministère de l'écologie, du développement durable et de l'énergie a entendu à la fois moderniser les objectifs (A) et se doter d'institutions plus performantes (B).

\footnotetext{
${ }^{4}$ Conformément aux articles $L$ 371-1 à $L$ 371-6 du Code de l'environnement.

5 Articles 27 à 31 du projet de loi.

6 Articles 67 à 72 du projet de loi.

Articles 37 à 68 du projet de loi.
} 


\section{A - LA MODERNISATION DES OBJECTIFS}

L'article $L$ 110-1 du Code de l'environnement qui vient qualifier d'intérêt général la protection de la biodiversité en France devrait faire l'objet d'un toilettage qui n'est pas anodin. D'abord, alors que cette disposition vient préciser que la qualification d'intérêt général porte sur la protection, la mise en valeur, la restauration, la remise en état et la gestion de ces espèces et habitats, le projet de loi vient préciser que relèvera désormais également de l'intérêt général «leur capacité à évoluer et la sauvegarde des services qu'ils fournissent ${ }^{8}$. Cette formulation comprend en réalité deux dimensions bien distinctes. La première, relative à la capacité d'évolution, permet de mieux s'inscrire dans l'esprit de la directive européenne habitats ${ }^{9}$ en considérant que la protection des espèces ne peut se limiter à assurer leur maintien en l'état, mais doit tenir compte de leur dynamique dans le temps et l'espace ${ }^{10}$.

Mais c'est surtout la référence aux services rendus par les espèces animales et végétales qui fait aujourd'hui l'objet des principales discussions. Ainsi la biodiversité n'est plus seulement défendue pour elle-même mais également compte tenu de son utilité parce qu'elle assure des services qui contribuent aux activités humaines, dit services écosystémiques. Cette approche s'appuie sur plusieurs études qui ont montré l'importance de la biodiversité qui, appréhendée en tant que source d'innovation, représente un capital économique extrêmement important. De fait, elle nourrit la recherche, de l'aile de la chauve souris pour l'aviation (biomimétisme) à l'exploitation des substances actives des plantes pour la pharmacie. Cependant, une telle approche qui a priori tend à faire mieux accepter les objectifs de préservation de la biodiversité présente également certains risques qu'il ne faut pas minimiser. En effet, il pourrait en découler une certaine hiérarchisation de la faune et la flore par un retour archaïque à la distinction entre espèces utiles à l'homme et les

\footnotetext{
${ }^{8}$ Article $2 \S 1^{\text {er }}$ du projet de loi.

9 Directive 92/43/CEE du Conseil du 21 mai 1992 concernant la conservation des habitats naturełs ainsi que de la faune et de la flore sauvage.

${ }^{10}$ Ainsi, it ressort de l'article 1er, sous i], de la directive habitats que l'état de conservation d'unes espèce animale est jugée favorable si «les données relatives à la dynamique de la population de l'espèce en question indiquent que cette espèce continue et est susceptible de continuer à long terme à constituer un élément viable des habitats naturels auxquels elle appartient» et que «l'aire de répartition naturelle de l'espèce ne diminue ni ne risque de diminuer dans un avenir prévisible» et qu'il «existe et il continuera probablement d'exister un habitat suffisamment étendu pour que ses populations se maintiennent à long terme».
} 
autres ${ }^{1.1}$. Sans doute, le scientifique n'aura pas de difficulté à considérer que toute espèce animale ou végétale répond à ce critère d'utilité en ce sens qu'il appartient à un maillon de la chaîne alimentaire, qu'il est un élément constitutif de tout un écosystème. Mais if n'est pas certain, par exemple, que le politique confronté à la protestation des éleveurs de moutons reconnaisse que le loup rend aussi de tels services! Différentes interprétations sont en effet possibles car s'il est question des services il n'est pas précisé à qui sont rendus ces services: seulement à l'homme ou à la nature, à la biodiversité en général?

Larticle L 110-1 du Code de l'environnement se trouve complété par une autre disposition non négligeable qui consiste à considérer d'intérêt général la connaissance de la nature ${ }^{12}$. Avec un peu de recul on peut s'étonner qu'une telle référence ne soit pas apparue plus tôt dans le Code de l'environnement dans la mesure où il relève du bon sens que de considérer que pour protéger il faut avant tout connaître. Pourtant, dans les faits, face à des projets d'aménagement, notamment à l'occasion des enquêtes d'utilité publique, on est souvent frappé par la pauvreté des avis délivrés par les services de l'Etat face à la richesse des apports fournis par certaines associations naturalistes sur le plan de la connaissance des enjeux environnementaux du site concerné. La raison de cette situation vient du fait que les politiques publiques souffrent, malgré des efforts réalisés ces dernières années, d'un véritable déficit en terme de connaissance des richesses naturelles du pays. Désormais le comblement de ces lacunes devrait relever de l'intérêt général. Encore faudra-t-il, pour cet objectif, de se doter des moyens nécessaires ce qui semble difficile dans un contexte de baisse du budget du ministère de l'environnement.

Par ailleurs, le paragraphe 2 de l'article L 110-1 $\$ 2$ du Code de l'environnement qui établit la liste des principes applicables en droit de l'environnement (précaution, prévention, pollueur-payeur, information et participation) devrait faire l'objet d'un double complément ${ }^{13}$. D'abord, l'alinéa 2 de ce paragraphe qui porte sur le «principe d'action préventive et de correction, par priorité à la source, des atteintes à l'environnement, en utilisant les meilleu-

11 Dans l'esprit de l'ancienne Convention de Paris du 19 mars 1902 sur la protection des oiseaux utiles à l'agriculture qui distinguait clairement les espèces méritant d'être protégées pour les services rendus aux activités agricoles de celles au contraire jugées nuisibles et pouvant faire l'objet d'actes de destruction!

12 Article $2 \S 1^{\text {er }}$ du projet de loi.

13 Article 2, $\S 2$ et 3 du projeet de loi. 
res techniques disponibles à un coût économiquement acceptable» devrait être complété par la formulation suivante: «ce principe implique d'éviter les atteintes à la biodiversité et, à défaut, d'en réduire la portée et de compenser les atteintes qui n'ont pu être évitées en tenant compte des fonctions écologiques de la biodiversité affectée». II s'agit là d'une consécration du principe de compensation qui supposerait, lorsque la prévention ne peut être évitée et donc la destruction de milieux naturel ne peut être empêchée par un projet, de tout mettre en œuvre pour rétablir un niveau de biodiversité équivalent. Enfin, un nouveau principe devrait également trouver sa place dans le Code de l'environnement. II s'agit du «principe de solidarité écologique qui appelle à prendre en compte, dans toute prise de décision publique ayant une incidence sur l'environnement, les interactions des écosystèmes, des êtres vivants et des milieux naturels ou aménagés».

Enfin, la dernière réforme d'ordre général vise à rappeler que dans la mise en œuvre de cette politique la France n'est pas isolée, mais s'inscrit dans le cadre de la Convention sur la biodiversité biologique du 5 juin 1992 . Cela explique qu'un nouvel article L110-3 est ajouté au Code de l'environnement afin de consacrer par la loi la mise en œuvre des stratégies nationales et régionales pour la biodiversité. II ne s'agit pas là d'une création ${ }^{14}$ mais plutôt de la consécration de ces stratégies en tant qu'instruments privilégiés pour assurer la cohérence des politiques publiques en matière de biodiversité.

\section{B - LE RENFORCEMENT DE L'EFFICACITÉ INSTITUTIONNELLE}

Au-delà des principes, le projet de loi vient renforcer l'outil institutionnel afin de lutter contre la multiplication des institutions. De fait, l'exposé des motifs accompagnant le projet de loi fait état de l'existence de pas moins de quarante-cinq organismes sous tutelle ou agréés et financés par l'Etat, agissant autour de la biodiversité, sans compter les parcs nationaux, parcs naturels régionaux, réserves naturelles. Un tel éclatement des compétences nuit de toute évidence à l'unité, la vision d'ensemble des actions mises en œuvre dans ce domaine.

L'efficacité passe donc par la création d'un nouvel établissement public, l'Agence française pour la biodiversité, présentée comme devant devenir

14 Voire l'actuelle stratégie nationale $2011-2020$ en cours. 
l'équivalent pour la biodiversité de ce qu'est l'ADEME ${ }^{15}$ pour la politique énergétique. L'objectif devrait être de renforcer la centralisation dans le but d'assurer une politique plus cohérente sur la préservation de la biodiversité, le développement des ressources ê services écosystémiques et la gestion équilibrée et durable des eaux. Cette agence a vocation à devenir un organe d'impulsion des politiques, notamment de la stratégie nationale pour la biodiversité ainsi que de soutien et de mise en réseau des différents acteurs.

Pour autant ces louables intentions se sont vite heurtées à des réalités politiques qui viennent largement vider le projet de son intérêt initial. En effet, la défense de leurs prérogatives par les organismes existant tels que l'office national des forêts et l'Office national de la chasse et de la faune sauvage qui n'intégreront pas l'Agence contrairement à ce qui avait été initialement prévu, a réduit largement la portée de la réforme. A défaut d'organiser leur fusion, le projet se réduirait à prévoir la signature de partenariats entre ces établissements et la nouvelle agence dont le budget prévisionnel a d'ailleurs déjà commencé à fondre puisque le budget initialement prévu de quatre cents millions d'euros annuels serait déjà divisé par deux ${ }^{16}$. Dans ces conditions on voit mal comment ce nouvel établissement public pourrait être en mesure de remplir effectivement l'ambitieuse mission qui lui est confiée.

\section{II - QUELQUES DISPOSITIONS TENDANT À FAVORISER UNE PRISE EN COMPTE EFFECTIVE DE LA BIODIVERSITÉ}

Au-delà des grands principes, le projet de loi prévoit un certain nombre de réformes plus précises. La principale tend à transposer en droit interne une série d'obligations auxquelles la France a souscrit en matière d'accès aux ressources génétiques (A). Par ailleurs, le projet de loi prévoit toute une série de nouveaux outils afin de renforcer l'action en matière de défense de la biodiversité $(B)$.

\footnotetext{
15 Agence pour le développement et la maîtrise de l'énergie.

${ }^{16}$ Louis de Redon, Le projet de loi-cadre sur la biodiversité à l'Assemblée nationale, Environnement $n^{\circ} 7$, Juillet 2014 , alerte 75 , et Louis de Redon, Un projet de loi-cadre sans surprise et des ordonnances à venir, Environnement $n^{\circ} 6$, Juin 2014.
} 


\section{A - L'ACCÈS AUX RESSOURCES GÉNÉTIOUES}

C'est sans doute le principal intérêt du projet qui vise à combler un véritable vide juridique: il s'agit de mettre en cuvre le protocole de Nagoya ${ }^{17}$ sur l'accès aux ressources génétiques signé par la France le 20 septembre $2011^{18}$. Rappelons que la Convention des Nations Unies sur la biodiversité biologique du 22 mai 1992 que le protocole vient compléter pose le principe de la conservation de la biodiversité dans le milieu naturel et dans les collections, l'utilisation durable de ces éléments et le partage juste et équitable des avantages issus de l'usage des ressources génétiques ${ }^{19}$.

Ainsi, il s'agit ici de revenir sur l'aspect utilitariste de la biodiversité. En effet, le patrimoine génétique est pris en compte en raison de son importance pour la recherche académique et surtout pour les filières pharmaceutiques, agro-alimentaires, cosmétique, biotechnologique ou horticoles. Compte tenu des enjeux il convient donc d'encadrer juridiquement l'accès à ces ressources génétiques en conciliant l'intérêt de la recherche, la sauvegarde de la souveraineté nationale sur ce patrimoine naturel et le respect des populations autochtones. L'objectif est d'organiser un régime d'accès et de partage des avantages tirés de ces ressources génétiques. Dans cet esprit les ressources ne seront accessibles, dans un certain nombre d'hypothèses, que si le bénéficiaire accepte un partage des connaissances et des services liés à ces recherches. Un chercheur ou une entreprise souhaitant utiliser une ressource génétique devra négocier le partage des avantages qu'il en tire afin d'obtenir un permis d'accès. II pourra s'agir soit de transfert de savoirs, afin par exemple de permettre l'accès à ces recherches sans intention de développement commercial dans des situations d'urgence menaçant la santé humaine, végétale ou animale, soit du versement de redevances qui seront alors versées au bénéfice de l'Agence française de la biodiversité.

\footnotetext{
i? Protocole de Nagoya du 29 octobre 2010 sur l'accès aux ressources génétiques et le partage juste et équitable des avantages découlant de leur utilisation relatif à la Convention sur la diversité biologique.

18 Articles 18 à 26 du projet de loi.

19 Selon l'article $1^{\text {er }}$ de la convention, «Les objectifs de la présente Convention, dont la réalisation sera conforme à ses dispositions pertinentes, sont la conservation de la diversité biologique, l'utilisation durable de ses éléments et le partage juste et équitable des avantages découlant de l'exploitation des ressources génétiques, notamment grâce à un accès satisfaisant aux ressources génétiques et à un transfert approprié des techniques pertinentes, compte tenu de tous les droits sur ces ressources et aux techniques, et grâce à un financement adéquat».
} 
Le système relevant de ce projet de loi se veut relativement souple afin de ne pas entraver la recherche. II devrait comprendre deux étapes. Les activités de recherche elles-mêmes ne feront l'objet que d'un régime déclaratif. Le régime d'autorisation quant à lui n'interviendra qu'au stade de la commer. cialisation des ressources génétiques et des connaissances traditionnelles associées. C'est à ce stade que le projet de loi prévoit le dispositif de partage qui se fera sur la base d'une large concertation pour définir ce qui est le plus pertinent à partager.

Enfin, conformément au protocole de Nagoya, ce régime suppose l'accord des communautés d'habitants qui détiennent des connaissances et des usages traditionnels. II faut en effet rappeler que c'est principalement outre- mer que la biodiversité se concentre, et à ce titre il convient de préserver les droits des populations autochtones des territoires d'outre-mer (notamment en Guyane, Nouvelle Calédonie...).

\section{B - LA CRÉATION DE NOUVEAUX OUTILS JURIDIQUES AU SERVICE DE LA BIODIVERSITÉ}

Enfin, sans révolutionner le droit de l'environnement, le projet de loi crée quelques outils supplémentaires qui peuvent se montrer utiles pour répondre aux objectifs généraux fixés par la loi. Cinq dispositions méritent ici d'être soulignées. La première porte sur l'élargissement du champ de compétence de la coopération intercommunale ${ }^{20}$. Si les Etablissements publics de coopération intercommunale détenaient déjà certaines compétences environnementales, ils pourront désormais être chargés «d'accroître et d'améliorer les connaissances sur l'environnement, leur diffusion, la sensibitisation et linformation des publics et d'assurer la conservation d'espèces ou la mise en place d'actions de restauration des milieux». C'est là une heureuse initiative que de permettre à cet échelon de proximité de mieux agir sur le respect de la bio. diversité, sous réserve cependant que l'Etat n'en profite pour se désinvestir partiellement sur ce terrain!

Dans un autre registre, la réforme ${ }^{21}$ autorise tout propriétaire de biens immobiliers à conclure des contrats avec un organisme public ou privé agis-

\footnotetext{
20 Article 32 du projet de loi.

21 Article 33 du projet de loi.
} 
Sant pour la protection de l'environnement (ONCFS ${ }^{22}$, Conservatoire du littoral, ONEMA ${ }^{23} \varepsilon$ ], contrats ayant pour finalité le maintien, la restauration de la biodiversité ou de services écosystémiques dans un espace naturel ou agricole en établissant des obligations réciproques. Surtout, un tel accord pourra faire naître à la charge des propriétaires successifs des obligations réelles que bon leur semble, l'accord en définissant le contenu, la durée et les modalités de résiliation. Lintérêt sera ici de permettre la mise en œuvre de mesures favo. rables à la biodiversité qui continueront à s'appliquer après le changement de propriétaire.

Ensuite, le nouvel article $L 411.2$ du Code de l'Environnement ${ }^{24}$ étend le champ d'application du régime des zones soumises à contraintes environnementales, dispositif qui n'existait jusqu'à présent que pour le maintien de la qualité de l'eau. Celui-ci autorise le préfet, sur des zones agricoles délimitées où il est nécessaire de maintenir ou restaurer un habitat, non seulement d'établir un programme d'actions pour restaurer ce milieu ${ }^{25}$, mais aussi et sur. tout de rendre obligatoire certaines pratiques agricoles favorables à l'espèce considérée ou à ses habitats, avec versement d'indemnités éventuelles pour les agriculteurs lorsque ces contraintes nouvelles impliqueraient des pertes de revenu. II s'agit ici de permettre de dépasser la seule logique contractuelle qui caractérise le plus souvent la gestion des sites Natura 2000. Ce mode de gestion présente en effet la faiblesse de faire dépendre l'efficacité des mesures de gestion de la bonne volonté des agriculteurs ou autres sylviculteurs. Ce nouveau dispositif, en cas d'absence de mise en œuvre de mesures concrètes faute d'accord des acteurs économiques concernés, devrait permettre au préfet d'imposer des modes de gestion afin d'assurer le respect des objectifs du cahier des charges de la zone Natura 2000 concernée.

Toujours dans le domaine agricole, le projet de loi prévoit également que le remembrement, jusqu'alors simple outil d'aménagement foncier agricole ou forestier devienne un instrument d'aménagement foncier agricole, forestier ou environnemental $\left.\right|^{26}$. L'élargissement du remembrement aux préoccupations environnementales devrait de façon tout à fait positive permettre d'agir contre

\footnotetext{
22 Office national de la chasse et de la faune sauvage.

${ }^{23}$ Office national de l'eau et des milieux aquatiques.

24 Article 34 du projet de loi.

2s Conformément à la procédure $L$ 114-1 du Code rural

25 Article 36 du projet de loi venant modifier l'article L. 123-1 du code rural et de la pêche maritime.
} 
l'éparpillement de certains sites protégés et apporter plus de cohérence à des zones naturelles.

Enfin, le projet de loi vient renforcer la logique punitive puisqu'il prévoit que les atteintes volontaires à la faune (principalement les actes de destruction] seront plus durement pénalisées. La peine maximale passe en effet de 15000 à 150000 euros, et de 150000 à 750000 si l'acte est commis en bande organisée 2 ? . Ce renforcement devrait avoir un louable effet dissuasif et devrait permettre d'éviter qu'un chasseur puisse «s'offrir» illégalement un loup ou un ours pour 15000 euros maximum comme la loi le permettait jusqu'ici. On regrettera cependant que la blessure de l'animal suite à un tir intentionnel ne soit pas prise en compte comme relevant de ces actes délictueux.

Enfin, même s'il s'agit d'une évidence, pour que ces nouveaux dispositifs puissent remplir pleinement leur rôle, faudra-t-il encore que les acteurs concernés (préfets, propriétaires fonciers, EPCI, juge pénal...) s'approprient pleinement ces réformes pour les mettre effectivement en œuvre. L'avenir de la biodiversité En France ne dépend donc pas du seul vote de ce projet de loi.

27 Article 52 du projet de loi. 